\title{
Fungal selective resorcylate aminopyrazole Hsp90 inhibitors: Optimization of whole cell anticryptococcal activity and insights into the structural origins of cryptococcal selectivity
}

Paul T. Marcyk, Emmanuelle V. LeBlanc, Douglas A. Kuntz, Alice Xue, Francisco Ortiz, Richard Trilles, Stephen Bengtson, Tristan M.G. Kenney, David S. Huang, Nicole Robbins, Noelle S. Williams, Damian J.

Krysan, Gilbert G. Privé*, Luke Whitesell*, Leah E. Cowen* and Lauren E. Brown*

* To whom correspondence should be addressed.

Emails: G.G.P.: Gil.Prive@ uhnresearch.ca; L.W.: luke.whitesell@ utoronto.ca;

L.E.C.: leah.cowen@utoronto.ca; L.E.B.: brownle@bu.edu

\section{Table of Contents}

Supplemental Table 1: Confirmation of lysate $\mathrm{EC}_{50 \mathrm{~S}}$ $\mathrm{S} 2$

Supplemental Figure S1: Correlation between lysate and purified NBD potencies in C. neoformans............. S3

Supplemental Table 2: Confirmation of fungal selectivity using purified NBDs ...................................... S4

Supplemental Figure S2: Correlation between lysate and purified NBD potencies in human ....................... S5

Supplemental Figure S3: Correlation between fold-selectivities calculated using lysates and purified NBDs. S6

Supplemental Table 3: HepG2 cytotoxicity dose-response data and statistics S7

Supplemental Table 4: NIH 3T3 cytotoxicity dose-response data and statistics ....................................... S8

Supplemental Table 5: Crystallographic data for apoprotein and NVP-AUY922-liganded structures ............ S9

Supplemental Table 6: Crystallographic data for RAP-liganded structures ......................................... S10

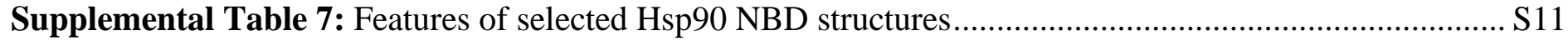

Supplemental Figure S4: HepG2 cytotoxicity dose-response curves .............................................. S12

Supplemental Figure S5: NIH 3T3 cytotoxicity dose-response curves ............................................. S13

Supplemental Figure S6: Ligand variability in the $C$. neoformans RAP complexes............................... S14

Supplemental Figure S7: Contact sites between ligands and structural elements of HSP90_...................... S14

Supplemental Table 8: Summary of computational docking results for Table 4 compounds...................... S15

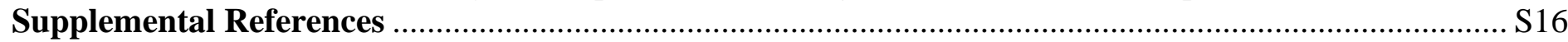


Supplemental Table 1. FP-based measurement in whole-cell lysates of relative Hsp90 binding affinity and fungal selectivity for resorcylate aminopyrazoles.

\begin{tabular}{|c|c|c|c|c|c|c|}
\hline \multirow[b]{2}{*}{ Compound } & \multicolumn{4}{|c|}{ C. neoformans } & \multicolumn{2}{|c|}{ HepG2 } \\
\hline & Potency $^{a}$ & SEM $^{\mathrm{b}}$ & Selectivity $^{c}$ & SEM $^{b}$ & Potency $^{\mathrm{a}}$ & SEM $^{\mathbf{b}}$ \\
\hline $9 d$ & 0.28 & 0.07 & 16.6 & 3.7 & 3.93 & 0.01 \\
\hline 9j & 0.14 & 0.04 & 10.2 & 2.9 & 1.17 & 0.12 \\
\hline 90 & 0.11 & 0.01 & 7.8 & 1.4 & 0.71 & 0.07 \\
\hline $9 v$ & 0.57 & 0.11 & 14.6 & 2.6 & 6.93 & 0.26 \\
\hline $9 \mathrm{ac}$ & 0.06 & 0.01 & 51.2 & 8.1 & 2.45 & 0.67 \\
\hline 9ad & 0.02 & 0.00 & 89.8 & 34.6 & 1.74 & 0.32 \\
\hline 9ae & 0.33 & 0.08 & 12.0 & 0.9 & 3.29 & 0.92 \\
\hline 9af & 0.14 & 0.02 & 27.9 & 1.7 & 3.28 & 0.36 \\
\hline 9ah & 0.28 & 0.02 & 8.9 & 2.2 & 2.11 & 0.39 \\
\hline 9ak & 0.40 & 0.01 & 7.9 & 0.9 & 2.65 & 0.22 \\
\hline 9am & 0.12 & 0.01 & 10.5 & 2.3 & 1.02 & 0.20 \\
\hline 9an & 0.12 & 0.02 & 21.9 & 1.8 & 2.15 & 0.37 \\
\hline 9ar & 0.30 & 0.05 & 11.8 & 2.8 & 2.98 & 0.49 \\
\hline $9 \mathrm{av}$ & 0.78 & 0.09 & $>15.3$ & 1.8 & $>10.00$ & 0.00 \\
\hline 9ax & 0.40 & 0.12 & 21.1 & 5.7 & 7.12 & 0.17 \\
\hline $9 \mathbf{a z}$ & 0.40 & 0.03 & 6.9 & 0.6 & 2.34 & 0.10 \\
\hline $9 \mathbf{b a}$ & 0.08 & 0.02 & 75.4 & 12.1 & 5.36 & 0.26 \\
\hline $9 \mathrm{bb}$ & 0.12 & 0.02 & 52.0 & 8.3 & 5.42 & 0.11 \\
\hline $9 b c$ & 0.25 & 0.03 & 12.9 & 1.4 & 2.77 & 0.12 \\
\hline $9 b d$ & 0.10 & 0.01 & 11.8 & 1.5 & 1.01 & 0.01 \\
\hline $9 b e$ & 0.29 & 0.03 & 22.9 & 1.9 & 5.63 & 0.65 \\
\hline $9 b f$ & 0.21 & 0.07 & 26.8 & 5.7 & 4.65 & 0.62 \\
\hline $9 b g$ & 1.08 & 0.04 & $>11.0$ & 0.3 & $>10.00$ & 0.00 \\
\hline $9 b i$ & 0.22 & 0.04 & 10.4 & 2.0 & 1.88 & 0.07 \\
\hline
\end{tabular}

\footnotetext{
${ }^{a}$ Mean $\mathrm{EC}_{50}(\mu \mathrm{M})$ : Concentration resulting in 50\% reduction in maximal polarization signal determined in 3 independent equilibrium competition binding experiments each consisting of duplicate determinations b Standard error of the mean

${ }^{\mathbf{c}}$ Geldanamycin-normalized mean ratio of $\mathrm{EC}_{50}$ values $(\mu \mathrm{M})$ determined in lysate of human HepG2 cells/indicated fungal species. Results are the mean of 3 independent experiments in each lysate type, each experiment consisting of duplicate determinations
} 


\section{Fungus: H99 Lysate $\mathrm{EC}_{50}$ vs. Purified H99 Hsp90 NBD $\mathrm{K}_{\mathrm{i}}$}

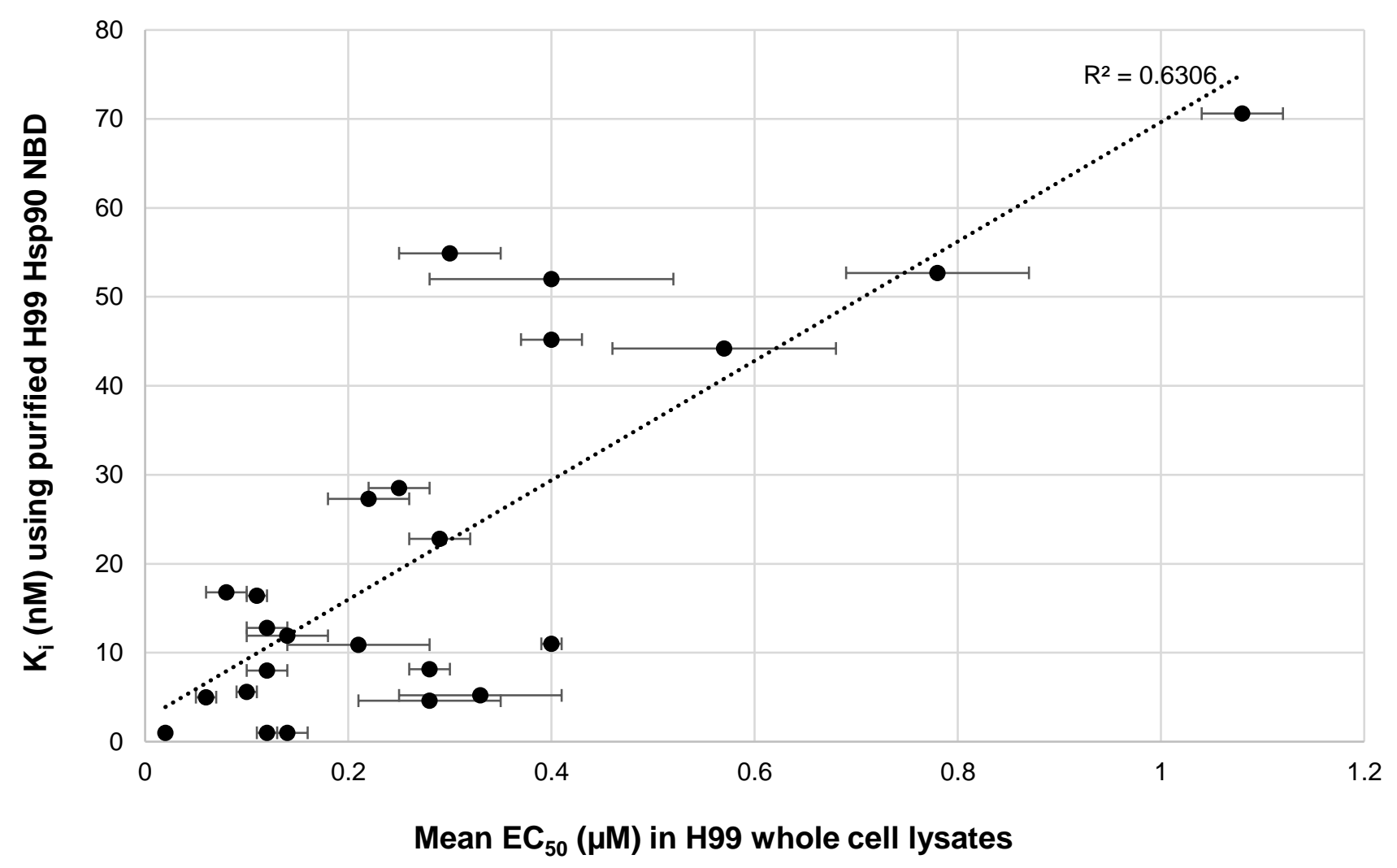

Supplemental Figure S1. Plot of compound potencies as determined in C. neoformans H99 whole cell lysates (mean $\mathrm{EC}_{50}$ values, $\mu \mathrm{M}$ ) vs $C$. neoformans $\mathrm{H} 99$ purified Hsp90 NBD ( $\mathrm{K}_{\mathrm{i}}$ values, $\mathrm{nM}$ ) for compounds appearing in Supplemental Tables 1 and 2. X-axis error bars depict standard error of the mean (SEM) for lysate $\mathrm{EC}_{50}$ determinations. Apparent potency, especially for lower affinity compounds is likely to be reduced in lysates compared to purified NBD due to the complex mixture of proteins present in lysate, some of which are likely to non-specifically adsorb compounds resulting in lower free concentrations. 
Supplemental Table 2. FP-based measurement using purified Hsp90 NBDs to determine relative Hsp90 binding affinity and fungal selectivity for resorcylate aminopyrazoles using $\mathrm{K}_{\mathrm{i}}$. Values for $\mathrm{K}_{\mathrm{i}}$ were determined as previously described. ${ }^{\text {1-S2 }}$

\begin{tabular}{|c|c|c|c|}
\hline Compound & $\begin{array}{c}\text { H99 Hsp90 } \\
\text { NBD Ki }(n M)\end{array}$ & 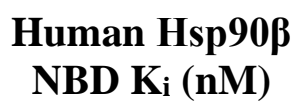 & $\begin{array}{c}\text { Fold } \\
\text { selectivity }\end{array}$ \\
\hline 9d & 4.6 & 145.4 & 31.4 \\
\hline 9j & 11.9 & 64.5 & 5.4 \\
\hline 90 & 16.4 & 146.9 & 9.0 \\
\hline $9 v$ & 44.2 & 976.4 & $>22.1$ \\
\hline $9 a c$ & 5.0 & 252.0 & 50.4 \\
\hline 9ad & $<1.0$ & 117.4 & $>117.1$ \\
\hline 9ae & 5.2 & 100.88 & 19.6 \\
\hline 9af & $<1.0$ & 118.1 & $>118.1$ \\
\hline 9ah & 8.15 & 132.70 & 16.3 \\
\hline 9ak & 11.0 & 136.9 & 12.5 \\
\hline 9am & $<1.0$ & 19.4 & $>19.4$ \\
\hline 9an & 12.8 & 243.5 & 19.1 \\
\hline 9ar & 54.9 & 617.6 & 11.3 \\
\hline $9 \mathrm{av}$ & 52.7 & $>997.9$ & $>18.9$ \\
\hline $9 \mathbf{a x}$ & 52.0 & $>976.2$ & $>18.8$ \\
\hline $9 \mathbf{a z}$ & 45.2 & 518.3 & 11.5 \\
\hline $9 \mathbf{b a}$ & 16.8 & 875.3 & 51.9 \\
\hline $9 \mathrm{bb}$ & 8.0 & 392.5 & 49.3 \\
\hline $9 b c$ & 28.5 & 791.8 & 27.8 \\
\hline 9bd & 5.6 & 56.3 & 10.1 \\
\hline $9 b e$ & 22.8 & 891.0 & 39.1 \\
\hline $9 b f$ & 10.9 & 260.8 & 23.9 \\
\hline $9 \mathrm{bg}$ & 70.6 & $>977.0$ & $>13.8$ \\
\hline 9bi & 27.3 & 670.6 & 24.5 \\
\hline
\end{tabular}




\section{Human: HepG2 Lysate $\mathrm{EC}_{50}$ vs. Purified Hsp90ß NBD $\mathrm{K}_{\mathrm{i}}$}

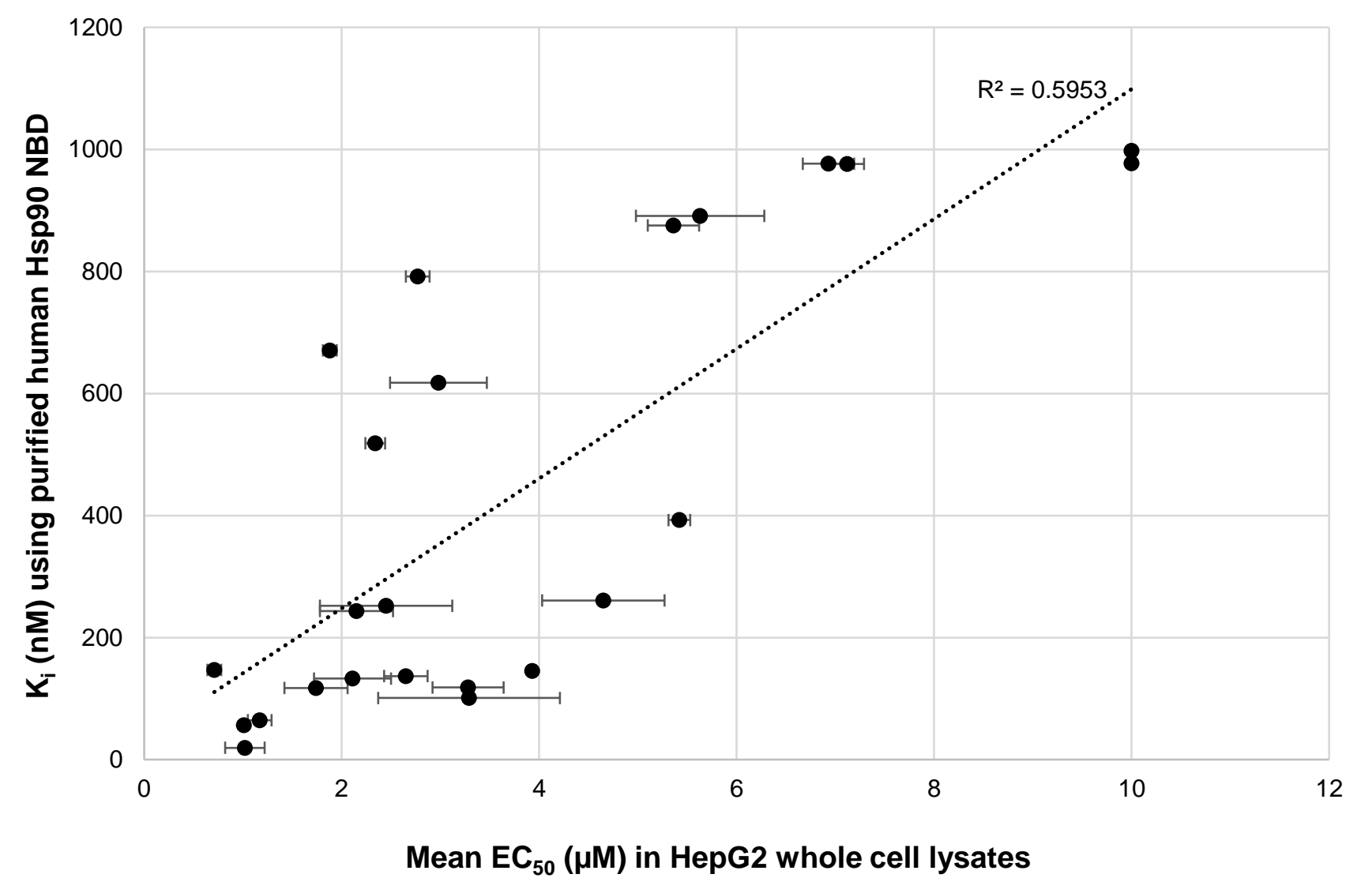

Supplemental Figure S2. Plot of compound potencies as determined in $\mathrm{HepG} 2$ whole cell lysates (mean $\mathrm{EC}_{50}$ values, $\mu \mathrm{M}$ ) vs purified human Hsp90 $\beta$ NBD $\left(\mathrm{K}_{\mathrm{i}}\right.$ values, $\mathrm{nM}$ ) for compounds appearing in Supplemental Tables 1-2. X-axis error bars depict standard error of the mean (SEM) for lysate $\mathrm{EC}_{50}$ determinations. Apparent potency, especially for lower affinity compounds is likely to be reduced in lysates compared to purified NBD due to the complex mixture of proteins present in lysate, some of which are likely to non-specifically adsorb compounds resulting in lower free concentrations. In addition, unlike $C$. neoformans, which expresses a single Hsp90 isoform, a mix of Hsp90 family isoforms (Hsp90a, Hsp90ß, Grp94 and Trap1) are present in human cell lysates, each of which contributes to tracer binding with a different intrinsic affinity. 
Fungal selectivity by lysate $\mathrm{EC}_{50}$ vs. purified NBD $\mathrm{K}_{\mathrm{i}}$

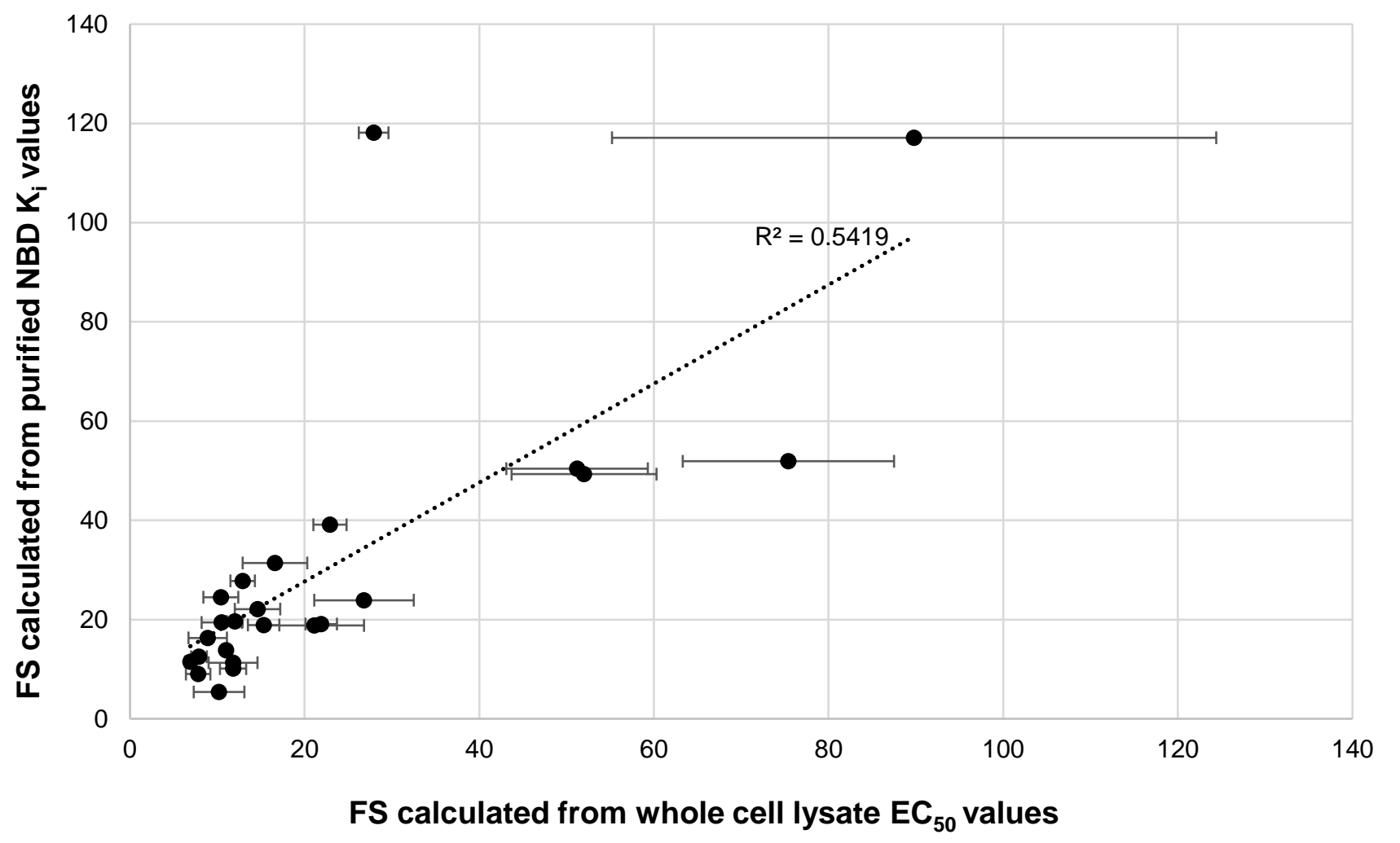

Supplemental Figure S3. Plot of cryptococcal fungal selectivities (FS) as calculated using whole cell lysate mean $\mathrm{EC}_{50}$ values (Supplemental Table 1) vs. purified NBD Ki values (Supplemental Table 2). X-axis error bars depict standard error of the mean (SEM) for lysate FS determinations. Unlike $C$. neoformans, which expresses a single Hsp90 isoform, a mix of Hsp90 family isoforms (Hsp90a, Hsp90ß, Grp94 and Trap1) are present in human cell lysates, each of which contributes to tracer binding with a different intrinsic affinity. 
Supplemental Table 3. Cytotoxicity for human liver-derived cancer cells. $\mathrm{IC}_{50}, 95 \% \mathrm{CI}$ and $\mathrm{R}^{2}$ values for select compounds exhibiting cryptococcal fold-selectivity $\geq 5$ and H99 MIC $80 \leq 12.5$ tested for cytotoxicity toward HepG2 cells in 9-point dose response (mean of triplicates).

$\begin{array}{cccc}\text { Compound } & \begin{array}{c}\text { HepG2 IC50 } \\ (\boldsymbol{\mu M})\end{array} & \text { 95\% } \mathbf{C I} & \mathbf{R}^{2} \\ \text { 9d } & 5.9 & 3.5 \text { to } 13.3 & 0.848 \\ \text { 9j } & 0.4 & 0.3 \text { to } 0.5 & 0.997 \\ \text { 9o } & 0.7 & 0.6 \text { to } 0.9 & 0.996 \\ \text { 9v } & 3.6 & 1.9 \text { to } 7.6 & 0.972 \\ \text { 9ac } & 0.3 & 0.2 \text { to } 0.4 & 0.992 \\ \text { 9ad } & 0.4 & 0.2 \text { to } 0.6 & 0.989 \\ \text { 9ae } & 0.5 & 0.4 \text { to } 0.6 & 0.998 \\ \text { 9af } & 0.4 & 0.3 \text { to } 0.6 & 0.987 \\ \text { 9ah } & 0.1 & 0.1 \text { to } 0.1 & 0.993 \\ \text { 9ak } & 0.5 & 0.4 \text { to } 0.7 & 0.995 \\ \text { 9am } & 0.3 & 0.3 \text { to } 0.3 & 0.998 \\ \text { 9an } & 0.9 & 0.8 \text { to } 2.0 & 0.979 \\ \text { 9ar } & 0.7 & 0.6 \text { to } 0.8 & 0.996 \\ \text { 9av } & 9.9 & 4.9 \text { to } 25.0 & 0.628 \\ \text { 9ax } & 11.3 & 6.9 \text { to } 13.3 & 0.977 \\ \text { 9az } & 2.2 & 2.2 \text { to } 2.7 & 0.998 \\ \text { 9ba } & 2.7 & 2.6 \text { to } 4.3 & 0.981 \\ \text { 9bb } & 0.6 & 0.4 \text { to } 1.0 & 0.978 \\ \text { 9bc } & 2.4 & 2.2 \text { to } 3.2 & 0.990 \\ \text { 9bd } & 0.8 & 0.7 \text { to } 0.9 & 0.997 \\ \text { 9be } & 1.9 & 1.8 \text { to } 3.0 & 0.980 \\ \text { 9bf } & 1.2 & 0.9 \text { to } 1.8 & 0.958 \\ \text { 9bg } & 3.1 & 1.1 \text { to } 8.6 & 0.879 \\ \text { 9bi } & 1.5 & 1.2 \text { to } 1.7 & 0.997 \\ & & & \end{array}$


Supplemental Table 4. Cytotoxicity for non-tumorigenic mouse fibroblasts. $\mathrm{IC}_{50}, 95 \% \mathrm{CI}$ and $\mathrm{R}^{2}$ values for select compounds exhibiting cryptococcal fold-selectivity $\geq 5$ and H99 MIC80 $\leq 12.5$ tested for cytotoxicity toward NIH 3 T3 cells in 9-point dose response (mean of triplicates).

$\begin{array}{cccc}\text { Compound } & \begin{array}{c}\text { NIH 3T3 } \\ \text { IC50 }(\boldsymbol{\mu M})\end{array} & \text { 95\% } \mathbf{C I} & \mathbf{R}^{\mathbf{2}} \\ \text { 9d } & 8.0 & 8.0 \text { to } 11.3 & 0.987 \\ \text { 9j } & 1.3 & 1.0 \text { to } 2.7 & 0.951 \\ \text { 9o } & 5.7 & 4.7 \text { to } 10.8 & 0.926 \\ \text { 9v } & 18.8 & 11.2 \text { to } 25.0 & 0.886 \\ \text { 9ac } & 2.8 & 2.2 \text { to } 3.7 & 0.989 \\ \text { 9ad } & 5.8 & 3.6 \text { to } 8.1 & 0.984 \\ \text { 9ae } & 1.9 & 1.1 \text { to } 3.9 & 0.965 \\ \text { 9af } & 5.8 & 4.3 \text { to } 10.1 & 0.939 \\ \text { 9ah } & 1.4 & 0.8 \text { to } 1.7 & 0.979 \\ \text { 9ak } & 4.4 & 3.0 \text { to } 6.8 & 0.972 \\ \text { 9am } & 1.8 & 0.3 \text { to } 4.4 & 0.977 \\ \text { 9an } & 2.9 & 2.3 \text { to } 3.7 & 0.993 \\ \text { 9ar } & 1.8 & 1.3 \text { to } 2.5 & 0.991 \\ \text { 9av } & -- & -- & 0.699 \\ \text { 9ax } & -- & -- & 0.855 \\ \text { 9az } & 4.6 & 2.5 \text { to } 12.8 & 0.921 \\ \text { 9ba } & 7.8 & 6.4 \text { to } 12.5 & 0.949 \\ \text { 9bb } & 7.1 & 4.9 \text { to } 9.4 & 0.874 \\ \text { 9bc } & 5.8 & 3.4 \text { to } 10.2 & 0.960 \\ \text { 9bd } & 1.4 & 0.9 \text { to } 2.3 & 0.983 \\ \text { 9be } & 3.1 & 0.6 \text { to } 10.3 & 0.867 \\ \text { 9bf } & 4.0 & 2.4 \text { to } 9.3 & 0.967 \\ \text { 9bg } & 11.2 & 7.2 \text { to } 19.3 & 0.967 \\ \text { 9bi } & 3.0 & 2.1 \text { to } 4.8 & 0.986\end{array}$

Note: ambiguous curve fit for $9 \mathbf{a v}$ and $9 \mathbf{a x}$ due to low toxicity 
Supplemental Table 5. Crystallographic data for apoprotein (PDB ID 7K9R) and NVP-AUY922-liganded (PDB ID 7K9S) structures

\begin{tabular}{|c|c|c|}
\hline ligand & Apo & NVP-AUY922 \\
\hline PDB ID & $7 \mathrm{~K} 9 \mathrm{R}$ & $7 \mathrm{~K} 9 \mathrm{~S}$ \\
\hline Beamline & APS 17_ID & NSLS_II AMX (17ID-1) \\
\hline Wavelength $(\AA)$ & 1.00 & 0.9202 \\
\hline Resolution range & $29.82-1.951(2.021-1.951)$ & $18.21-3.21(3.324-3.21)$ \\
\hline Space group & C 121 & P 1211 \\
\hline Unit cell $\left(\AA,^{\circ}\right)$ & $66.2380 .26149 .89,9092.390$ & $51.6478 .45188 .11,9095.790$ \\
\hline Total reflections & 209487 (17925) & $243235(11500)$ \\
\hline Unique reflections & $48718(4717)$ & $24421(2323)$ \\
\hline Multiplicity & $4.3(3.8)$ & $5.2(4.8)$ \\
\hline Completeness (\%) & $85.22(83.38)$ & $98.65(96.43)$ \\
\hline Mean I/ $\sigma(\mathrm{I})$ & $16.76(3.14)$ & $6.04(1.29)$ \\
\hline R-merge & $0.03001(0.333)$ & $0.096(0.323)$ \\
\hline R-meas & $0.04244(0.471)$ & $0.1219(0.826)$ \\
\hline $\mathrm{CC} 1 / 2$ & $0.999(0.71)$ & $0.996(0.65)$ \\
\hline Reflections used in refinement & $48711(4716)$ & $24400(2322)$ \\
\hline Reflections used for R-free & $1783(175)$ & $1216(111)$ \\
\hline R-work & $0.1685(0.2564)$ & $0.2123(0.3204)$ \\
\hline R-free & $0.2011(0.3026)$ & $0.2510(0.3386)$ \\
\hline Number of non-hydrogen atoms & 5470 & 10139 \\
\hline Protein chains / ligand molecules & $3 / 0$ & $6 / 6$ \\
\hline Protein residues & 634 & 1270 \\
\hline solvent atoms & 414 & 0 \\
\hline Bond RMSD (A) & 0.01 & 0.005 \\
\hline Angle RMSD $\left(^{\circ}\right)$ & 1.18 & 0.74 \\
\hline Ramachandran favored (\%) & 97.45 & 96.33 \\
\hline Ramachandran allowed (\%) & 2.39 & 3.35 \\
\hline Ramachandran outliers (\%) & 0.16 & 0.32 \\
\hline Rotamer outliers (\%) & 1.97 & 2.75 \\
\hline Average $B$-factor & 34.99 & 83.3 \\
\hline macromolecules & 34.62 & 83.3 \\
\hline ligands & & 83.45 \\
\hline solvent & 39.55 & \\
\hline
\end{tabular}


Supplemental Table 6. Crystallographic data for RAP-liganded structures containing 19 (7K9U), 18 (7K9V), and $10(7 \mathrm{~K} 9 \mathrm{~W})$.

\begin{tabular}{|c|c|c|c|}
\hline ligand & 19 & 18 & 10 \\
\hline PDB ID & $7 \mathrm{~K} 9 \mathrm{U}$ & $7 \mathrm{~K} 9 \mathrm{~V}$ & $7 \mathrm{~K} 9 \mathrm{~W}$ \\
\hline Beamline & NSLS_II FMX (17ID-2) & NSLS_II FMX (17ID-2) & NSLS_II FMX (17ID-2) \\
\hline Wavelength $(\AA)$ & 0.978 & 0.97932 & 0.97932 \\
\hline Resolution range & $29.33-1.925(1.993-1.925)$ & $29.29-1.91(1.978-1.91)$ & $29.48-2.13(2.19-2.13)$ \\
\hline Space group & C 121 & C 121 & C 121 \\
\hline Unit cell $\left(\AA,{ }^{\circ}\right)$ & $145.1244 .3377 .44,90103.890$ & 145.5644 .297890104 .490 & $145.5044 .9577 .54,90103.690$ \\
\hline Total reflections & $243837(22932)$ & $244880(20590)$ & $179,020(10232)$ \\
\hline Unique reflections & $36323(3444)$ & $37103(3568)$ & $27266(1770)$ \\
\hline Multiplicity & $6.7(6.7)$ & $6.6(5.8)$ & $6.6(5.8)$ \\
\hline Completeness (\%) & $98.84(94.08)$ & $98.16(96.25)$ & $98.6(83.9)$ \\
\hline Mean $I / \sigma(I)$ & $16.74(2.42)$ & $18.17(2.45)$ & $9.20(1.30)$ \\
\hline R-merge & $0.06207(0.8155)$ & $0.01647(0.1998)$ & $0.0319(0.353)$ \\
\hline R-meas & $0.06765(0.8837)$ & $0.02329(0.2825)$ & $0.04512(0.4992)$ \\
\hline $\mathrm{CC} 1 / 2$ & $0.998(0.905)$ & $0.999(0.939)$ & $0.999(0.791)$ \\
\hline Reflections used in refinement & $36274(3433)$ & $37086(3565)$ & $27266(2458)$ \\
\hline Reflections used for R-free & $1357(139)$ & $1390(140)$ & $1015(87)$ \\
\hline R-work & $0.2111(0.3553)$ & $0.1658(0.2362)$ & $0.1862(0.2635)$ \\
\hline R-free & $0.2340(0.4124)$ & $0.1948(0.2964)$ & $0.2286(0.2932)$ \\
\hline Number of non-hydrogen atoms & 3449 & 3651 & 3393 \\
\hline Protein chains / ligand molecules & $2 / 2$ & $2 / 2$ & $2 / 2$ \\
\hline Protein residues & 414 & 755 & 534 \\
\hline solvent atoms & 199 & 340 & 116 \\
\hline Bond RMSD $(\AA)$ & 0.011 & 0.006 & 0.007 \\
\hline Angle RMSD $\left(^{\circ}\right)$ & 1.2 & 0.78 & 0.87 \\
\hline Ramachandran favored (\%) & 98.77 & 98.04 & 97.07 \\
\hline Ramachandran allowed (\%) & 1.23 & 1.72 & 2.69 \\
\hline Ramachandran outliers (\%) & 0 & 0.25 & 0.24 \\
\hline Rotamer outliers (\%) & 2.37 & 0 & 0.89 \\
\hline Average B-factor & 50.63 & 49.63 & 55.29 \\
\hline macromolecules & 50.74 & 49.53 & 55.48 \\
\hline ligands & 39.46 & 41.05 & 50.53 \\
\hline solvent & 52.35 & 52.87 & 53.78 \\
\hline
\end{tabular}


Supplemental Table 7. Features of selected Hsp90 NBD structures

\begin{tabular}{|c|c|c|c|c|c|c|}
\hline Species & Ligand & PDB ID & $\begin{array}{l}\text { Fused } \alpha 4- \\
\alpha 5 ?\end{array}$ & $\begin{array}{l}\text { Disrupted } \\
\text { L93/F124/W148 } \\
\text { core? }^{\mathrm{c}}\end{array}$ & $\begin{array}{l}\text { Disrupted } \\
\text { W148/F156 } \\
\text { stack? }^{c}\end{array}$ & $\begin{array}{l}\text { Disrupted } \\
\beta 1 \text { and } \\
\text { shifted } \alpha 1 ?\end{array}$ \\
\hline $\begin{array}{l}C . \\
\text { neoformans }\end{array}$ & (apo) & $7 \mathrm{~K}^{2} \mathrm{R}^{\mathrm{a}}$ & No & No & No & No \\
\hline $\begin{array}{l}C . \\
\text { neoformans }\end{array}$ & AUY922 & $7 \mathrm{~K} 9 \mathrm{~S}^{\mathrm{a}}$ & Yes/No ${ }^{b}$ & No & No & No \\
\hline $\begin{array}{l}C . \\
\text { neoformans }\end{array}$ & 19 & $7 \mathrm{~K} 9 \mathrm{U}^{\mathrm{a}}$ & Yes & Yes & Yes & Yes \\
\hline $\begin{array}{l}C . \\
\text { neoformans }\end{array}$ & 18 & $7 \mathrm{~K} 9 \mathrm{~V}^{\mathrm{a}}$ & Yes & Yes & Yes & Yes \\
\hline $\begin{array}{l}\text { C. } \\
\text { neoformans }\end{array}$ & 10 & $7 \mathrm{~K}^{2} \mathrm{~W}^{\mathrm{a}}$ & Yes & Yes & Yes & Yes \\
\hline C. albicans & (apo) & $6 \mathrm{CJI}$ & No & No & No & No \\
\hline C. albicans & ADP & $6 \mathrm{CJJ}$ & No & No & No & No \\
\hline C. albicans & AUY922 & 6CJS & Yes & No & Yes & Yes \\
\hline C. albicans & radicicol & $6 \mathrm{CJL}$ & No & No & No & No \\
\hline C. albicans & SNX-2112 & $6 \mathrm{CJR}$ & No & Yes & No & No \\
\hline C. albicans & CMLD013075 & $6 \mathrm{CJP}$ & No & No & Yes & Yes \\
\hline Human & (apo) & $\begin{array}{l}\text { 1YER, } \\
\text { 1YES }\end{array}$ & No & No & No & No \\
\hline Human & AUY922 & $2 \mathrm{VCI}$ & No & No & No & No \\
\hline Human & radicicol & 4EGK & No & No & No & No \\
\hline Human & SNX-2112 & $4 \mathrm{NH} 7$ & Yes & Yes & No & No \\
\hline Human & PU3 & 1UY6 & Yes & Yes & No & No \\
\hline Human & AT13387 & $2 \mathrm{XJX}$ & No & No & No & No \\
\hline
\end{tabular}

${ }^{\mathrm{a}}$ This work

${ }^{\mathrm{b}}$ The helix is fused in two of the six chains.

${ }^{\mathrm{c}}$ C. neoformans numbering. C. neoformans residues L93, F124, W148 and F156 are equivalent to C. albicans L96, F127, W151, F159 and human L107, F138, W162 and F170. 


\section{HepG2 cytotoxicity}
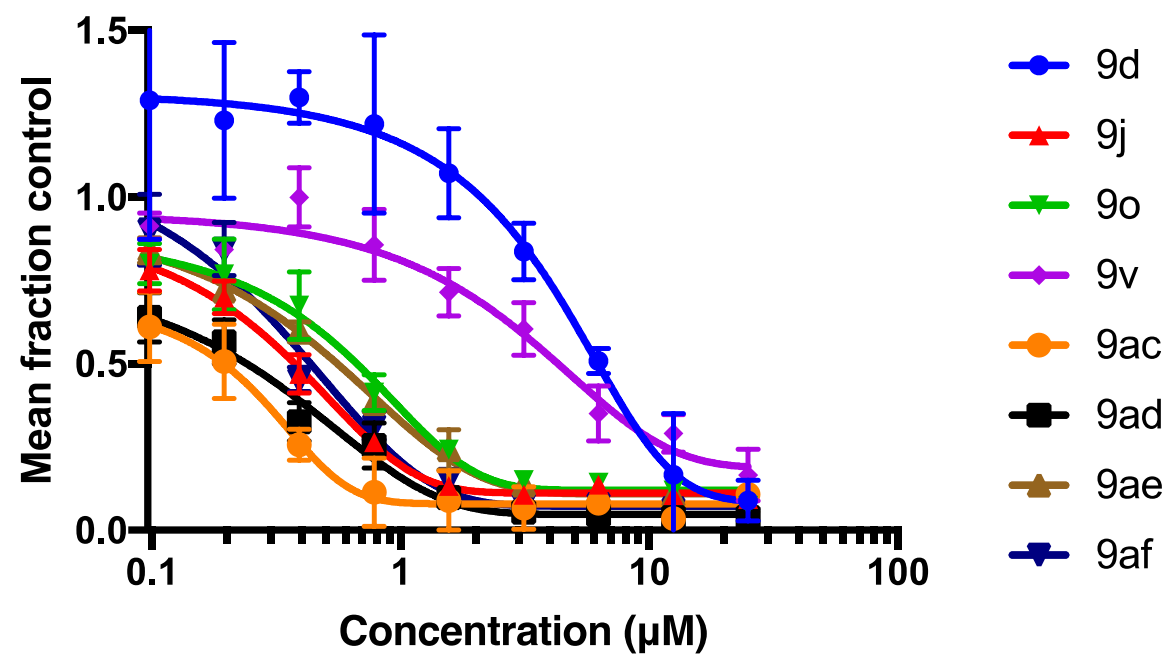

HepG2 cytotoxicity
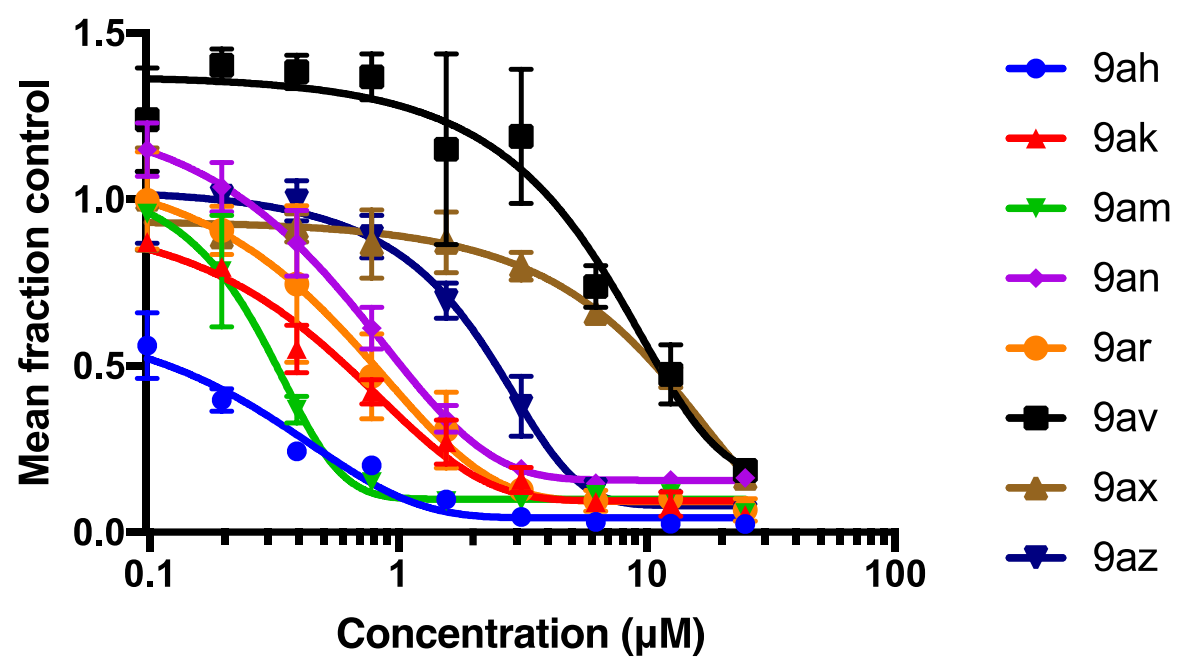

HepG2 cytotoxicity

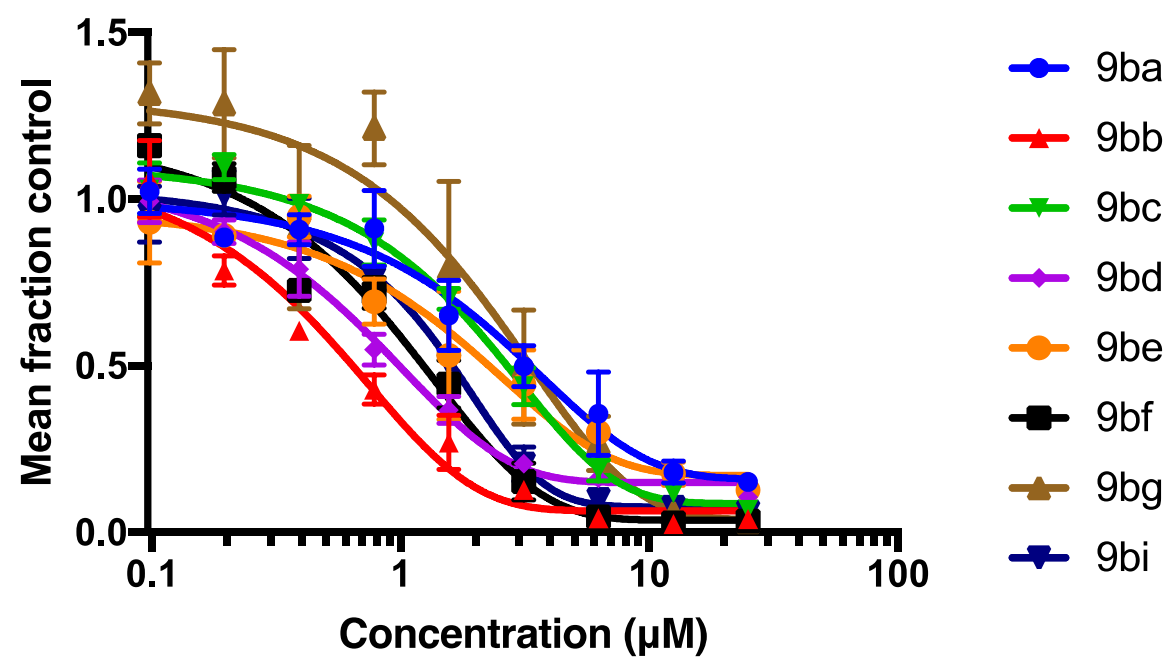

Supplemental Figure S4. HepG2 cytotoxicity curves for select compounds, including all compounds exhibiting cryptococcal fold-selectivity $\geq 5$ and $\mathrm{H}_{99} \mathrm{MIC}_{80} \leq 12.5$. 


\section{T3 cytotoxicity}

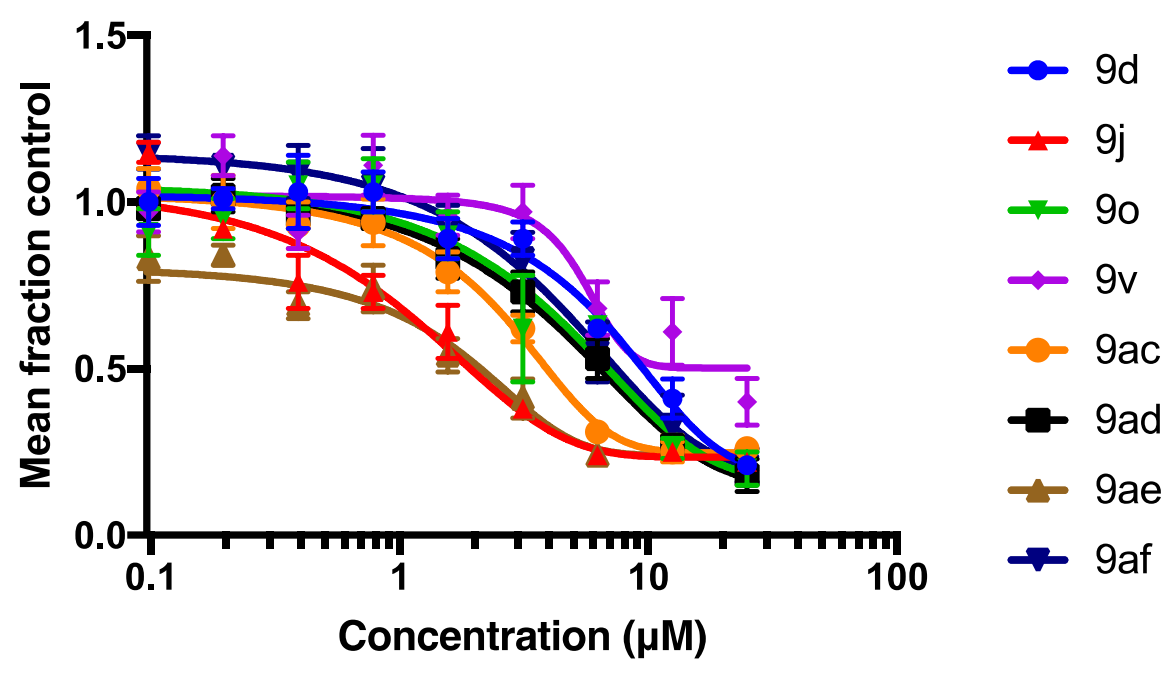

3T3 cytotoxicity

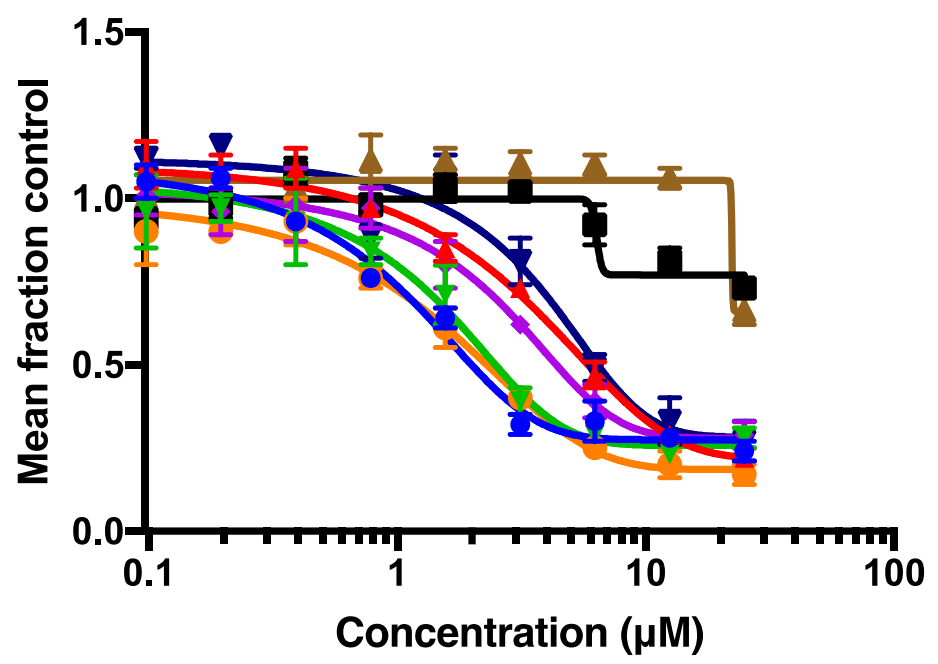

$\rightarrow$ 9ah

$+9 a k$

$\rightarrow$ 9am

$\sim 9$ an

- 9ar

- 9av

$-9 a x$

$\rightarrow-9 a z$

3T3 cytotoxicity

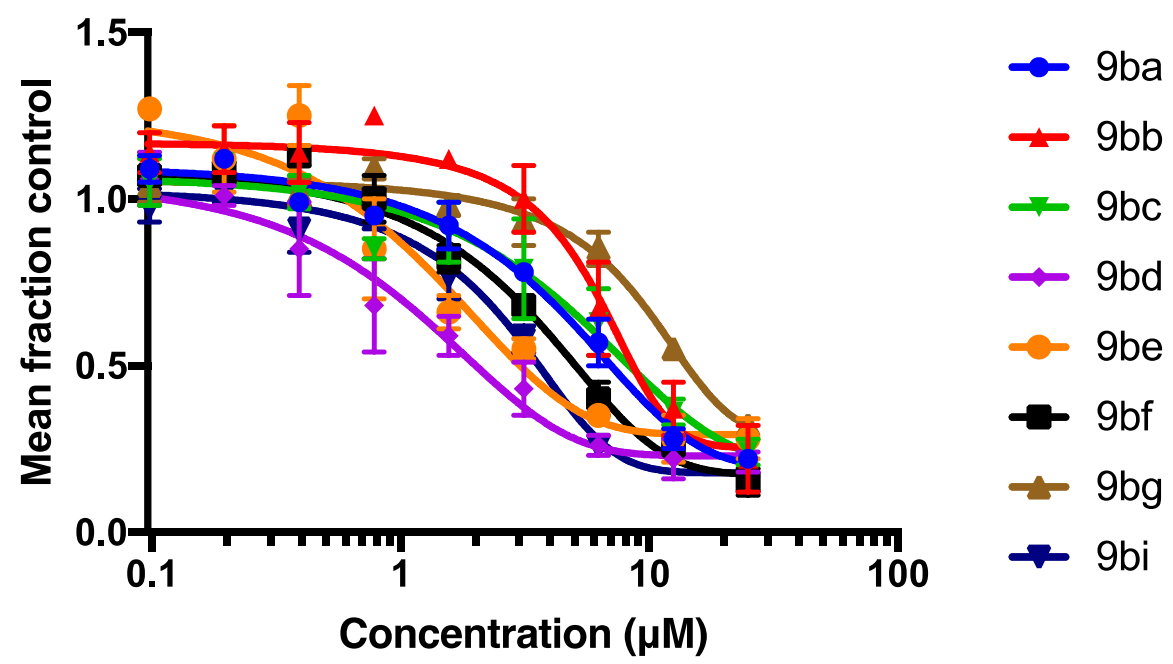

Supplemental Figure S5. NIH 3T3 (3T3) cytotoxicity curves for select compounds, including all compounds exhibiting cryptococcal fold-selectivity $\geq 5$ and $\mathrm{H} 99 \mathrm{MIC}_{80} \leq 12.5$. 
A

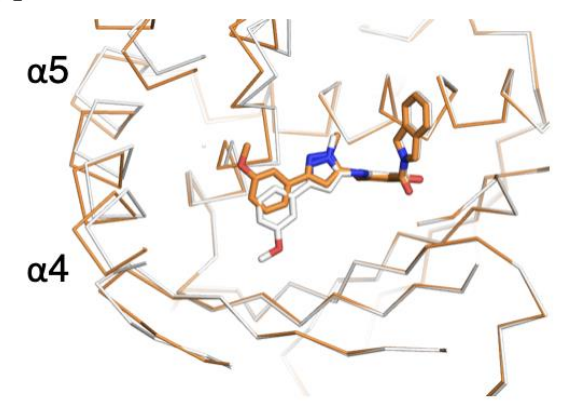

B

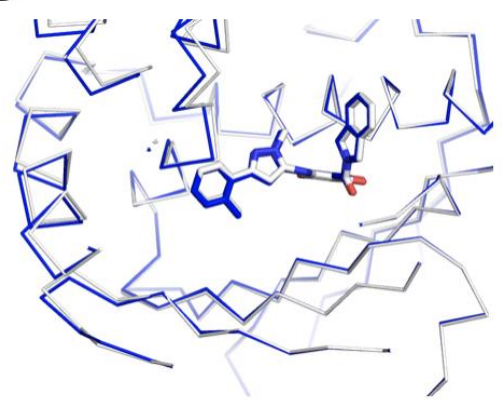

C

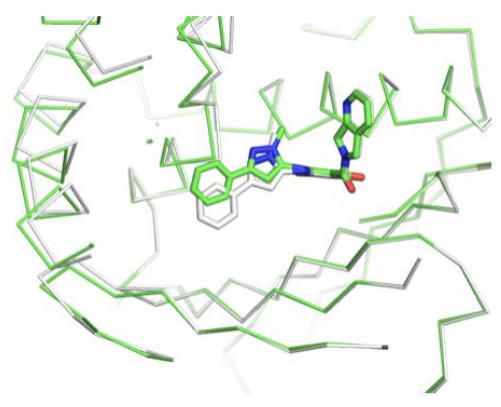

Supplemental Figure S6. Ligand variability in the $C$. neoformans RAP complexes. The two crystallographically independent chains in each structure are overlaid, with the carbons of one chain colored gray. Complexes with compounds 10 (PDB 7K9W), 18 (PDB 7K9V) and $\mathbf{1 9}$ (PDB 7K9U) are shown in panels $\mathbf{A}, \mathbf{B}$ and $\mathbf{C}$, respectively.
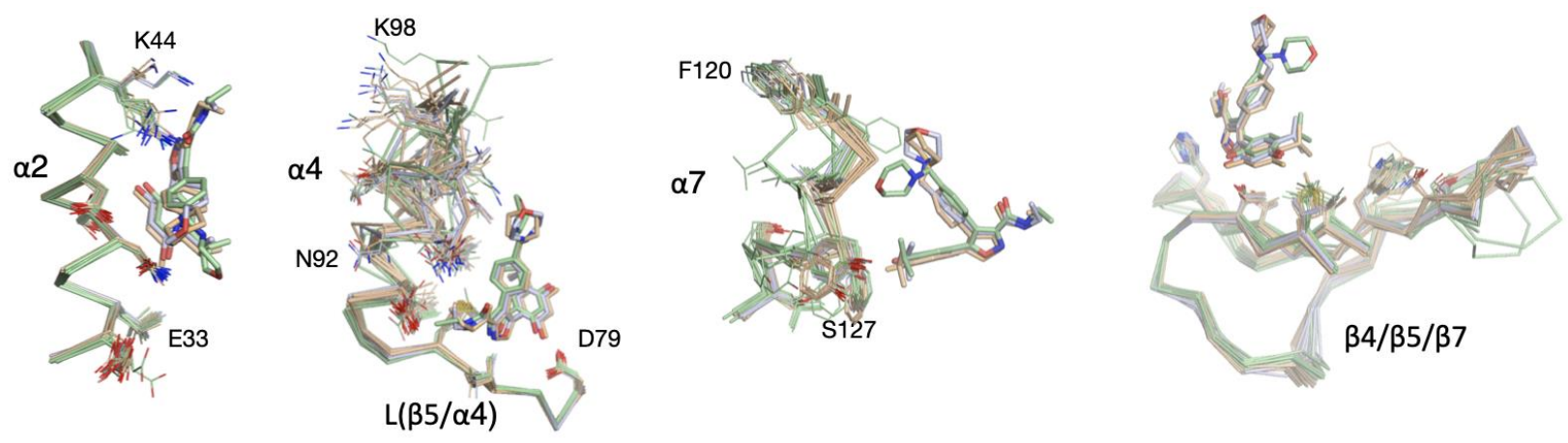

Supplemental Figure S7. Contact sites between the ligands and the different structural elements of Hsp90. All complexes and apo structures from Fig. 1 are shown (32 chains, PDB IDs 1YER, 1YES, 4EGK, 4NH7, 2VCI, 2XJX, 1UY6, 6CJI, 6CJJ, 6CJL, 6CJR, 7K9R, 7K9S, 7K9U, 7K9V, 7K9W, 6CJS, 6CJP) and selected side chains are indicated. $\mathrm{C} \alpha$ traces are shown in orange, green and pale blue for $C$. neoformans $(7 \mathrm{~K} 9 \mathrm{R}, 7 \mathrm{~K} 9 \mathrm{~S}$, 7K9U, 7K9V, 7K9W), C. albicans (6CJI, 6CJJ, 6CJL, 6CJR, 6CJS, 6CJP) and human (1YER, 1YES, 4EGK, 4NH7, 2VCI, 2XJX, 1UY6) structures, respectively, and selected side chains are indicated. 
Supplemental Table 8. Summary of computational docking results for Table 4 compounds. Every docking pose comported to one of the two $\mathrm{R}^{2}$ rotamers observed for inhibitors $\mathbf{1 0}$ and 18, which hold the $\mathrm{R}^{2}$ aryl ring within $35^{\circ}$ of co-planarity with the pyrazole ring. The respective dihedrals measured for the top rotamer type I pose (red) and/or rotamer type II pose (blue) as indicated below for each compound into each docking grid are reported. Also reported are each compound's best docking score, and the average \pm standard deviation of the scores for all poses in each row. For each compound, the dihedral(s) corresponding to the topscored pose(s) are underlined.
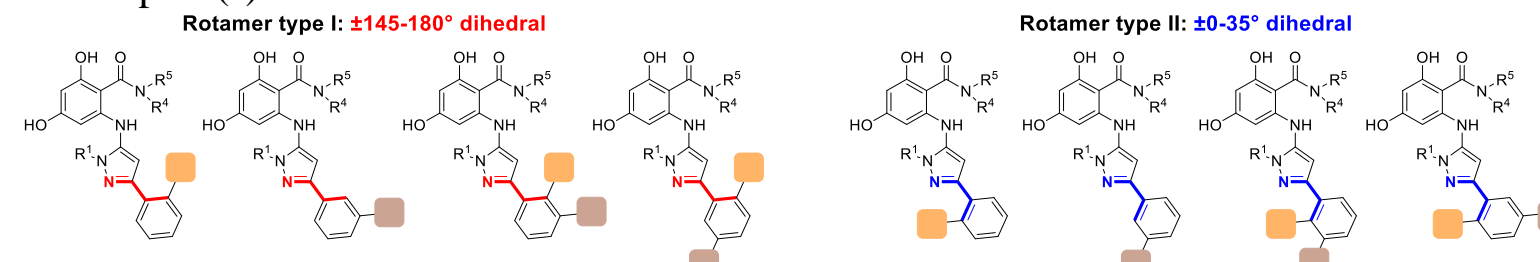

\begin{tabular}{|c|c|c|c|c|c|c|c|c|c|c|}
\hline \multirow[b]{2}{*}{ Compound } & \multirow[b]{2}{*}{$\mathbf{R}^{\mathbf{1}}$} & \multirow[b]{2}{*}{$\mathbf{R}^{2}$} & \multicolumn{6}{|c|}{ Rotamer Dihedrals for Best Type I and Type II Poses } & \multicolumn{2}{|c|}{ Docking Scores } \\
\hline & & & $\begin{array}{c}\text { 7K9U } \\
\text { Chain } \\
\text { A }\end{array}$ & $\begin{array}{c}7 \mathrm{~K} 9 \mathrm{U} \\
\text { Chain } \\
\text { B }\end{array}$ & $\begin{array}{c}\text { 7K9V } \\
\text { Chain } \\
\text { A }\end{array}$ & $\begin{array}{c}\text { 7K9V } \\
\text { Chain } \\
\text { B }\end{array}$ & $\begin{array}{c}7 \mathrm{~K} 9 \mathrm{~W} \\
\text { Chain } \\
\mathbf{A}\end{array}$ & $\begin{array}{c}7 \mathrm{~K} 9 \mathrm{~W} \\
\text { Chain } \\
\text { B }\end{array}$ & $\begin{array}{c}\text { Best } \\
\text { Score } \\
\text { (kcal/mol) }\end{array}$ & $\begin{array}{c}\text { Avg } \\
\text { Score } \\
\text { (kcal/mol) }\end{array}$ \\
\hline $\begin{array}{l}\text { Native } \\
\text { Ligand }\end{array}$ & $\mathrm{Me}$ & $(\mathrm{va}$ & $N / A$ & $N / A$ & $\begin{array}{c}153^{\circ} \\
-- \\
\end{array}$ & $\begin{array}{c}154^{\circ} \\
--\end{array}$ & $\begin{array}{c}- \\
-19^{\circ} \\
\end{array}$ & $\begin{array}{c}162^{\circ} \\
--\end{array}$ & $N / A$ & $N / A$ \\
\hline 9d & $t-\mathrm{Bu}$ & & $\begin{array}{c}160^{\circ} \\
--\end{array}$ & $\begin{array}{l}168^{\circ} \\
-10^{\circ}\end{array}$ & $\frac{155^{\circ}}{--}$ & $\begin{array}{l}152^{\circ} \\
-32^{\circ}\end{array}$ & $\begin{array}{l}165^{\circ} \\
-19^{\circ}\end{array}$ & $\begin{array}{c}164^{\circ} \\
--\end{array}$ & -14.4 & $\begin{array}{c}-12.6 \pm \\
0.7\end{array}$ \\
\hline $9 \mathbf{j}$ & CyHex & & $\begin{array}{c}\text { No } \\
\text { poses }\end{array}$ & $\begin{array}{c}\text { No } \\
\text { poses }\end{array}$ & $\begin{array}{c}\text { No } \\
\text { poses }\end{array}$ & $\begin{array}{c}\text { No } \\
\text { poses }\end{array}$ & $\begin{array}{c}\text { No } \\
\text { poses }\end{array}$ & $\begin{array}{c}\text { No } \\
\text { poses }\end{array}$ & $N / A$ & $N / A$ \\
\hline 90 & $t$-Bu & & $\begin{array}{c}158^{\circ} \\
-- \\
\end{array}$ & $-\overline{-}$. & $\frac{152^{\circ}}{--}$ & $\begin{array}{c}145^{\circ} \\
-- \\
\end{array}$ & $-\overline{-}$ - & $\begin{array}{c}167^{\circ} \\
-- \\
\end{array}$ & -14.7 & $\begin{array}{l}-12.6 \\
\pm 0.9 \\
\end{array}$ \\
\hline $9 v$ & $t-\mathrm{Bu}$ & & $\begin{array}{l}163^{\circ} \\
-18^{\circ} \\
\end{array}$ & $\begin{array}{l}174^{\circ} \\
-12^{\circ} \\
\end{array}$ & $\frac{155^{\circ}}{-20^{\circ}}$ & $\begin{array}{l}150^{\circ} \\
-23^{\circ} \\
\end{array}$ & $\begin{array}{l}166^{\circ} \\
-11^{\circ} \\
\end{array}$ & $\begin{array}{l}163^{\circ} \\
-17^{\circ} \\
\end{array}$ & -14.4 & $\begin{array}{l}-12.5 \\
\pm 0.9 \\
\end{array}$ \\
\hline 9am & $\mathrm{Me}$ & & $\begin{array}{l}166^{\circ} \\
-20^{\circ}\end{array}$ & $\begin{array}{c}171^{\circ} \\
-6^{\circ}\end{array}$ & $\frac{158^{\circ}}{-28^{\circ}}$ & $\begin{array}{l}154^{\circ} \\
-31^{\circ}\end{array}$ & $\begin{array}{l}166^{\circ} \\
-18^{\circ}\end{array}$ & $\begin{array}{l}160^{\circ} \\
-16^{\circ}\end{array}$ & -15.5 & $\begin{array}{l}-13.5 \\
\pm 0.8\end{array}$ \\
\hline 9an & $\mathrm{Me}$ & & $\begin{array}{l}161^{\circ} \\
-29^{\circ}\end{array}$ & $\begin{array}{l}171^{\circ} \\
-11^{\circ}\end{array}$ & ${\frac{153^{\circ}}{-30^{\circ}}}^{\circ}$ & $\begin{array}{l}152^{\circ} \\
-35^{\circ}\end{array}$ & $\begin{array}{l}163^{\circ} \\
-20^{\circ}\end{array}$ & $\begin{array}{l}159^{\circ} \\
-17^{\circ}\end{array}$ & -15.3 & $\begin{array}{l}-13.5 \\
\pm 0.9\end{array}$ \\
\hline 9ac & $\mathrm{Me}$ & & $\begin{array}{l}172^{\circ} \\
-20^{\circ} \\
\end{array}$ & $\begin{array}{l}176^{\circ} \\
-19^{\circ} \\
\end{array}$ & ${\frac{152^{\circ}}{--}}^{\circ}$ & $\begin{array}{c}155^{\circ} \\
-- \\
\end{array}$ & $\begin{array}{c}162^{\circ} \\
-- \\
\end{array}$ & $\begin{array}{c}162^{\circ} \\
-- \\
\end{array}$ & -15.5 & $\begin{array}{l}-13.7 \\
\pm 0.9 \\
\end{array}$ \\
\hline 9ad & $\mathrm{Me}$ & & $\begin{array}{c}164^{\circ} \\
--\end{array}$ & $\begin{array}{l}169^{\circ} \\
-11^{\circ}\end{array}$ & ${\frac{152^{\circ}}{--}}^{\circ}$ & $\begin{array}{c}153^{\circ} \\
--\end{array}$ & $\begin{array}{l}161^{\circ} \\
-23^{\circ}\end{array}$ & $\begin{array}{c}162^{\circ} \\
--\end{array}$ & -15.4 & $\begin{array}{l}-13.3 \\
\pm 0.9 \\
\end{array}$ \\
\hline 9ae & $\mathrm{Me}$ & & $\begin{array}{l}164^{\circ} \\
-21^{\circ} \\
\end{array}$ & $\begin{array}{l}174^{\circ} \\
-10^{\circ} \\
\end{array}$ & ${\frac{155^{\circ}}{--}}^{\circ}$ & $\begin{array}{c}153^{\circ} \\
--\end{array}$ & $\begin{array}{c}161^{\circ} \\
--\end{array}$ & $\begin{array}{c}161^{\circ} \\
--\end{array}$ & -15.5 & $\begin{array}{l}-13.6 \\
\pm 1.0 \\
\end{array}$ \\
\hline $9 a x$ & $t$-Bu & & $\begin{array}{c}160^{\circ} \\
--\end{array}$ & $\begin{array}{l}167^{\circ} \\
-11^{\circ} \\
\end{array}$ & ${\frac{159^{\circ}}{-25^{\circ}}}^{\circ}$ & $\begin{array}{l}156^{\circ} \\
-32^{\circ} \\
\end{array}$ & $\begin{array}{l}166^{\circ} \\
-19^{\circ} \\
\end{array}$ & $\begin{array}{c}165^{\circ} \\
--\end{array}$ & -14.9 & $\begin{array}{l}-12.9 \\
\pm 0.8 \\
\end{array}$ \\
\hline $9 a z$ & CyHex & & $\begin{array}{c}\text { No } \\
\text { poses }\end{array}$ & $\begin{array}{c}\text { No } \\
\text { poses }\end{array}$ & $\begin{array}{c}\text { No } \\
\text { poses }\end{array}$ & $\begin{array}{c}\text { No } \\
\text { poses }\end{array}$ & $\begin{array}{c}\text { No } \\
\text { poses }\end{array}$ & $\begin{array}{c}\text { No } \\
\text { poses }\end{array}$ & $N / A$ & $N / A$ \\
\hline 9ah & $\mathrm{Me}$ & & $-21^{\circ}$ & $\begin{array}{c}\text { No } \\
\text { poses }\end{array}$ & ${\frac{155^{\circ}}{-29}}^{\circ}$ & $\begin{array}{l}154^{\circ} \\
-29^{\circ}\end{array}$ & $-\overline{-1}^{\circ}$ & $\begin{array}{c}159^{\circ} \\
--\end{array}$ & -15.0 & $\begin{array}{l}-13.8 \\
\pm 0.8\end{array}$ \\
\hline $9 \mathrm{ba}$ & $i-\operatorname{Pr}$ & & $-3^{\circ}$ & $\begin{array}{c}\text { No } \\
\text { poses }\end{array}$ & ${\frac{155^{\circ}}{-30^{\circ}}}^{\circ}$ & $\begin{array}{l}149^{\circ} \\
-30^{\circ}\end{array}$ & $-\overline{-}^{\circ}$ & $\begin{array}{c}161^{\circ} \\
--\end{array}$ & -14.7 & $\begin{array}{l}-13.4 \\
\pm 0.8\end{array}$ \\
\hline $9 b b$ & $t$-Bu & & $-21^{\circ}$ & $\begin{array}{c}\text { No } \\
\text { poses }\end{array}$ & ${\frac{154^{\circ}}{--}}^{\circ}$ & $\begin{array}{l}156^{\circ} \\
-27^{\circ}\end{array}$ & $-\overline{12}{ }^{\circ}$ & $\begin{array}{c}162^{\circ} \\
--\end{array}$ & -14.3 & $\begin{array}{l}-12.6 \\
\pm 0.9\end{array}$ \\
\hline $9 b c$ & CyPent & & $\begin{array}{c}\text { No } \\
\text { poses }\end{array}$ & $\begin{array}{c}\text { No } \\
\text { poses }\end{array}$ & $\begin{array}{l}146^{\circ} \\
\underline{-37}^{\circ}\end{array}$ & $\begin{array}{c}\text { No } \\
\text { poses }\end{array}$ & $\begin{array}{c}\text { No } \\
\text { poses }\end{array}$ & $\begin{array}{c}162^{\circ} \\
--\end{array}$ & -14.4 & $\begin{array}{l}-13.9 \\
\pm 0.5\end{array}$ \\
\hline 9bd & CyHex & & $\begin{array}{c}\text { No } \\
\text { poses }\end{array}$ & $\begin{array}{c}\text { No } \\
\text { poses }\end{array}$ & $\begin{array}{c}\text { No } \\
\text { poses }\end{array}$ & $\begin{array}{c}\text { No } \\
\text { poses }\end{array}$ & $\begin{array}{c}\text { No } \\
\text { poses }\end{array}$ & $\begin{array}{c}\text { No } \\
\text { poses }\end{array}$ & $N / A$ & $N / A$ \\
\hline 9av & $t$-Bu & & $\begin{array}{c}156^{\circ} \\
--\end{array}$ & $\begin{array}{c}\text { No } \\
\text { poses }\end{array}$ & ${\frac{153^{\circ}}{-31^{\circ}}}^{\circ}$ & $\begin{array}{l}150^{\circ} \\
-28^{\circ}\end{array}$ & $-\overline{2}^{\circ}$ & $\begin{array}{c}166^{\circ} \\
--\end{array}$ & -14.2 & $\begin{array}{l}-12.6 \\
\pm 1.0\end{array}$ \\
\hline
\end{tabular}




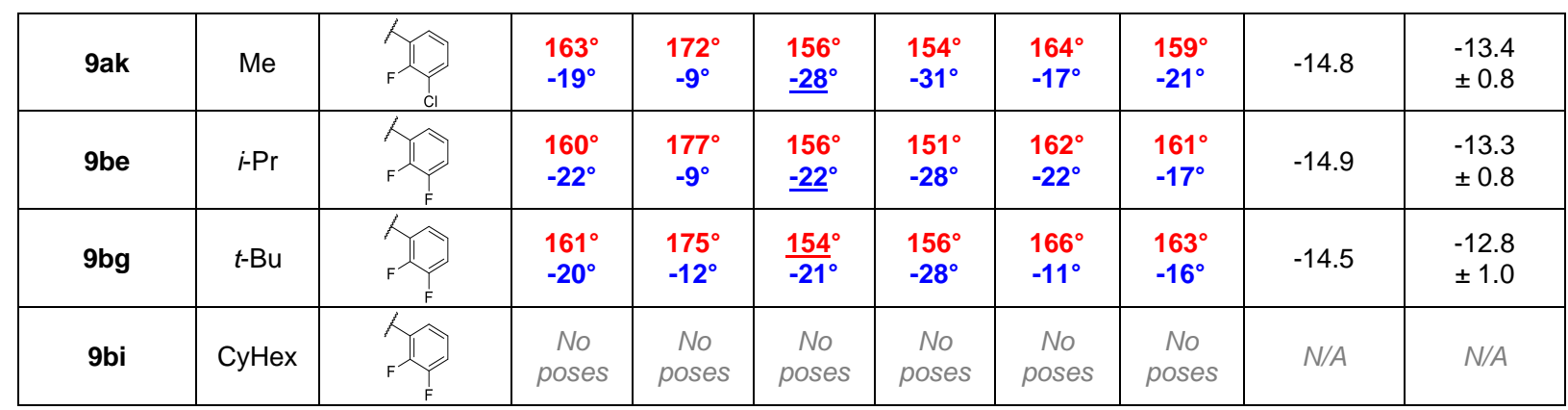

\section{Supplemental References.}

S1. Rossi, A. M.; Taylor, C. W., Analysis of protein-ligand interactions by fluorescence polarization. Nat. Protoc. 2011,6(3), 365-387.

S2. Whitesell, L.; Robbins, N.; Huang, D. S.; McLellan, C. A.; Shekhar-Guturja, T.; LeBlanc, E. V.; Nation, C. S.; Hui, R.; Hutchinson, A.; Collins, C.; Chatterjee, S.; Trilles, R.; Xie, J. L.; Krysan, D. J.; Lindquist, S.; Porco, J. A.; Tatu, U.; Brown, L. E.; Pizarro, J.; Cowen, L. E., Structural basis for species-selective targeting of Hsp90 in a pathogenic fungus. Nat. Commun. 2019,10(1), 402. 